\title{
Conocimientos sobre salud sexual y su relación con el comportamiento sexual en estudiantes universitarios
}

\section{Knowledge on sexual health and its relationship with sexual behavior in university students}

\author{
Joe Ordoñez Sánchez ${ }^{1, a}$, Jhony Real Cotto ${ }^{1, b}$, Jefferson Gallardo León ${ }^{1, c}$, Hugo Alvarado Franco ${ }^{2, d}$, \\ Aurora Roby Arias ${ }^{3, e}$ \\ ${ }^{1}$ Docente, Universidad Nacional de Guayaquil, Ecuador \\ ${ }^{2}$ Docente Titular de la Universidad Técnica de Babahoyo, Ecuador \\ ${ }^{3}$ Médico internista, Hospital Neumológico "Alfredo J. Valenzuela", Ecuador \\ ${ }^{a}$ Magister en Gerencia en Salud; ${ }^{b}$ Magister en Epidemiologia; c Magister en Gerencia en Servicios de Salud; d Especialista en Ginecología y Obstetricia, Doctor en Medicina \\ y Cirugia; e Magister en Salud Pública. Especialista en Medicina Interna. Doctor en Medicina y Cirugía.
}

\section{Correspondencia Jhony Real Cotto Telef. $0997202031 ; 042214198$ Direcc. Urbanización Polaris, Mz. 797 villa 22. Guayaquil- Ecuador. jreal_cotto@hotmail.com; realcottoj@gmail.com}

Recibido: 20 de abril 2017. Aceptado: 1 de junio 2017.

Conflicto de interés: el autor declara que no tener conflicto de interés y el contenido del manuscrito no ha sido publicado previamente.

Consentimiento informado: aprobado por La Facultad de Ciencias de la Salud - Universidad Técnica de Babahoyo.

Fuente de financiamiento: autofinanciado.

Citar como: Ordoñez Sánchez J, Real Cotto J, Gallardo León J, Alvarado Franco $\mathrm{H}$, Roby Arias A. Conocimientos sobre salud sexual y su relación con el comportamiento sexual en estudiantes universitarios. An Fac med. 2017;78(4):419-23 DOI: http://dx.doi.org/10.15381/ anales.v78i4.14264
An Fac med. 2017;78(4):419-23 / http://dx.doi.org/10.15381/anales.v78i4.14264

\section{Resumen}

Introducción. La salud sexual y reproductiva es de gran importancia y se define como un proceso ligado al desarrollo humano; es decir, comienza desde el nacimiento y se desarrolla durante toda la vida. Objetivo: Establecer el conocimiento sobre salud sexual y su relación con el comportamiento sexual de los estudiantes en las edades de 18 a 25 años de la Facultad de Ciencias de la Salud de la Universidad Técnica de Babahoyo, año 2014. Diseño: no experimental, transversal, de tipo descriptivo comparativo. Lugar: Facultad de Ciencias de la Salud de la Universidad Técnica de Babahoyo de la Provincia de Los Ríos, Ecuador. Participantes: Estuvo conformada por 140 estudiantes de las escuelas de obstetricia y enfermería. Intervenciones: Se utilizó entrevista directa cara a cara, utilizando un cuestionario validado del Proyecto Integrado: "La salud sexual y la salud reproductiva de la población estudiantil de la Universidad Nacional de Costa Rica". Medidas de resultados: Para el análisis se empleó los indicadores descriptivos y analíticos entre las variables. Resultados: La mayoría de los estudiantes (92\%) se declara como persona sexualmente activa, mientras que el $76 \%$ ha practicado sexo oral. En cuanto a la actitud el $50 \%$ de los estudiantes está en desacuerdo en que los hombres que se arreglan más sean afeminados mientras que el $81 \%$ desaprueba que las mujeres que no se preocupan por su apariencia sean marimachos. Conclusiones: Los estudiantes en su mayoría se declaran sexualmente activos y tres de cada cuatro estudiantes practican el sexo oral, presentando un nivel medio en conocimientos y comportamiento.

Palabras clave: Salud sexual; Conocimiento; Comportamiento

\section{Abstract}

Introduction: Sexual and reproductive health is of great importance and is defined as a process linked to human development; That is, it begins at birth and develops throughout life. Objective: To establish the knowledge about sexual health and its relationship with sexual behavior of students in the ages of 18 to 25 years of the Faculty of Health Sciences of the Technical University of Babahoyo, year 2014. Design: non-experimental, transversal, comparative descriptive type. Place: Faculty of Health Sciences of the Technical University of Babahoyo Province of Los Ríos, Ecuador. Participants: Comprised 140 students from the schools of obstetrics and nursing. Interventions: A direct face-to-face interview using a validated questionnaire of the Integrated Project: "Sexual health and reproductive health of the student population of the National University of Costa Rica" was used. Measures of results: For the analysis the descriptive and analytical indicators between the variables were used. Results: The majority of students (92\%) declared themselves to be sexually active, while $76 \%$ had oral sex. As for the attitude $50 \%$ of the students disagree that the men who are more adjusted are effeminate while $81 \%$ disapprove that women who do not care about their appearance are tomboy. Conclusions: Students are mostly sexually active and three out of four students practice oral sex, presenting a medium level of knowledge and behavior.

Keywords: Sexual health; Knowledge; Behaviors 


\section{INTRODUCCIÓN}

La educación sobre salud sexual es responsabilidad de toda una sociedad, iniciándose desde el nacimiento dentro del grupo familiar y transcurre durante toda la vida en los sucesivos vínculos que establecen las personas al interactuar con diferentes grupos. Actualmente, se aprecian importantes cambios de conductas, actitudes y valores en lo referente a la sexualidad de los jóvenes y adolescentes de distintos sexos ${ }^{(1)}$.

La adolescencia y la juventud son etapas de la vida en el que las conductas de riesgo son en parte las causas que originan lesiones, trastornos de salud, secuelas y en oportunidades hasta la muerte en ambos sexos (2); además, es en esta etapa donde se van definiendo la mayor parte de las prácticas que determinan las opciones y estilos de vida en las que se sustentan la autoconstrucción de la salud. Considerar que la vulnerabilidad del adolescente y joven está en función de su historia anterior, sumada a la susceptibilidad o fortalezas inducidas por los cambios de algunos factores sociales, demográficos, el microambiente familiar, las políticas socioeconómicas, así como el nivel de conocimiento que poseen sobre Salud Sexual y Reproductiva.

Es importante el tomar medidas por parte de la familia para cultivar las funciones que como organismo social le incumben, y se pueda enfrentar en el hogar la labor educativa, específicamente la que se refiere al desarrollo de la educación sexual ${ }^{(3)}$.Por esta razón, debe crearse un medio ambiente favorable en el cual cada persona pueda disfrutar de sus derechos sexuales como parte del proceso de desarrollo. Si bien las personas experimentan su sexualidad en formas que varían dependiendo de factores internos y externos, los derechos humanos relacionados con la sexualidad, su protección y promoción deberían, en todo el mundo, formar parte de la existencia diaria de todas las personas. Los derechos sexuales son derechos humanos universales que se basan en la libertad, la dignidad e igualdad inherentes a todos los seres humanos ${ }^{(4)}$.

Dentro del Modelo de Atención Integral de Salud del Ministerio de Salud
Pública del Ecuador ${ }^{(5)}$, la salud sexual y reproductiva es de gran importancia y se define como un proceso ligado al desarrollo humano; es decir, comienza desde el nacimiento y se desarrolla durante toda la vida; asimismo, educa a mujeres a llevar una vida reproductiva sana y libre de riesgos, así como su sexualidad y en forma responsable ${ }^{(6)}$.

Bajo esta nueva visión; "El Ministerio de Salud Pública, ejercerá plenamente la gobernanza del Sistema Nacional de Salud, con un modelo referencial en Latinoamérica que priorice la promoción de la salud y la prevención de enfermedades, con altos estándares de calidad y calidez de atención, garantizando la salud integral de la población y el acceso universal a una red de servicios, contando con la participación coordinada de organizaciones públicas, privadas y de la comunidad" (7); así pues, la salud sexual y reproductiva se convierte en un proceso social de construcción de valores y de comportamientos individuales, familiares y comunitarios; de creación de una nueva cultura en el cuidado de la salud y del desarrollo institucional, acorde con el desarrollo humano y con la corresponsabilidad social de los individuos, la comunidad y las instituciones.

Es oportuno considerar que los adolescentes y jóvenes obtienen información no adecuada y toman decisiones dentro del contexto de la cultura en que viven confrontando problemas sociales. En el departamento de bienestar estudiantil de la universidad técnica de Babahoyo existe un grupo profesional de apoyo frente a los problemas que presentan los estudiantes, respecto a las conductas que asumen los jóvenes provenientes de hogares disfuncionales, de padres que ocultan el tema de sexo por protección a los hijos o que viven con otras personas, lo que conlleva a una desinformación responsable del comportamiento sexual; estas acciones no son suficientes para evitar los problemas de salud que surgen con este comportamiento inadecuado.

En la Facultad de Ciencias de la Salud de la Universidad Técnica de Babahoyo no existe una asignatura de educación sexual y solo se imparte ciertos conocimientos no integrados como anatomía y fisiología del aparato reproductor sobre salud sexual. El presente estudio es de relevancia social, potencial teórico-práctico y mejoramiento sobre la salud sexual que proporciona orientación hacia un rediseño de la estructura curricular de la Facultad de Ciencias de la Salud, optimizando la calidad de vida de sus estudiantes para prevenir las consecuencias de los escasos conocimientos sobre salud sexual. Su objetivo es establecer los conocimientos sobre salud sexual y su relación con su comportamiento sexual de los estudiantes en las edades de 18 a 25 años de la Facultad de Ciencias de la Salud de la Universidad Técnica de Babahoyo durante el año 2014.

\section{MÉTODOS}

La investigación fue no experimental, transversal, de tipo descriptivo comparativo ${ }^{(8,9)}$. La muestra estuvo conformada por 140 estudiantes de la Facultad de Ciencias de la Salud de la cuidad de Babahoyo, durante el año 2014. Las unidades de análisis estuvieron conformadas por los estudiantes de las Escuelas de Obstetricia (89) y de Enfermería (51). Utilizando como instrumento el cuestionario en el que se identifican conocimientos que los estudiantes tienen sobre educación en salud sexual y las características de sus respectivos comportamientos sexuales.

Se incluyeron a estudiantes que estaban matriculados en las escuelas de enfermería y obstetricia de la Universidad Técnica de Babahoyo durante el 2014, y accedieron a colaborar; se excluyeron a estudiantes que proceden de traslado interno o de otras universidades del país y que no tengan matrículas consecutivas.

Se respetaron los principios éticos, se salvaguardó la integridad y privacidad de los participantes ya que no se tomó ningún nombre o referencia alguna de las personas entrevistadas, usando códigos al procesar y reportar los resultados; además, se solicitó el consentimiento informado a cada uno de ellos.

La técnica utilizada fue la entrevista directa cara a cara, para evaluar los conocimientos y comportamiento sobre salud sexual en los estudiantes de las escuelas académicas de la Facultad de Ciencias de la Salud. Se utilizó un cuestionario 
Tabla 1. Características socio culturales de los estudiantes.

\begin{tabular}{|c|c|c|c|}
\hline \multirow[t]{2}{*}{ Variables } & & No. & Porcentaje \\
\hline & Total & 140 & $100 \%$ \\
\hline \multirow[t]{4}{*}{ Grupo etario } & $18-19$ & 30 & 21,4 \\
\hline & $20-21$ & 45 & 32,1 \\
\hline & $22-23$ & 45 & 32,1 \\
\hline & $24-25$ & 20 & 14,2 \\
\hline \multirow[t]{2}{*}{ Género } & Masculino & 37 & 26 \\
\hline & Femenino & 103 & 74 \\
\hline \multirow[t]{5}{*}{ Estado civil } & Solteros & 110 & 79 \\
\hline & Casados & 9 & 6 \\
\hline & Unión libre & 14 & 10 \\
\hline & Separado & 6 & 4 \\
\hline & Divorciado & 1 & 0,7 \\
\hline \multirow[t]{4}{*}{ Número de hijos } & Sin hijos & 110 & 79 \\
\hline & Uno & 23 & 16 \\
\hline & Dos & 4 & 3 \\
\hline & Más de dos & 3 & 2 \\
\hline \multirow[t]{7}{*}{ Religión que profesa } & Evangélica & 18 & 13 \\
\hline & Pentecostal & 3 & 2 \\
\hline & Católica & 55 & 39 \\
\hline & Testigo de Jehová & 24 & 17 \\
\hline & Mormona & 9 & 6 \\
\hline & Otras & 2 & 1,4 \\
\hline & Ateos & 25 & 18 \\
\hline \multirow[t]{4}{*}{ Religiosidad } & Muy religioso & 14 & 10 \\
\hline & Regularmente religioso & 66 & 47 \\
\hline & Poco religioso & 39 & 28 \\
\hline & Nada religioso & 21 & 15 \\
\hline
\end{tabular}

Fuente: Encuesta a estudiantes de obstetricia y enfermería.

Tabla 2. Conocimientos sobre salud sexual.

\begin{tabular}{lcc}
\hline Afirmaciones verdaderas & No. & Porcentaje \\
\hline $\begin{array}{l}\text { En una relación sexual es importante saber qué le gusta a la pareja. } \\
\begin{array}{l}\text { La salud sexual incluye comprender las consecuencias de las acciones que } \\
\text { se tomen }\end{array}\end{array}$ & 2 & $9 \%$ \\
$\begin{array}{l}\text { La salud sexual incluye la promoción de la igualdad entre hombres y } \\
\text { mujeres }\end{array}$ & 24 & $13 \%$ \\
\hline $\begin{array}{l}\text { La salud sexual incluye distinguir entre los deseos personales y los de las } \\
\text { otras personas }\end{array}$ & 128 & $92 \%$ \\
$\begin{array}{l}\text { Afirmaciones falsas } \\
\text { La sexualidad está limitada a la genitalidad }\end{array}$ & 43 & $31 \%$ \\
\hline $\begin{array}{l}\text { El acto sexual es mejor cuando se toma licor } \\
\text { La sexualidad es sólo lo biológico o anatómico }\end{array}$ & 8 & $6 \%$ \\
\hline $\begin{array}{l}\text { El acto sexual es mejor cuando se consumen drogas } \\
\text { Los hombres que tienen un pene más grande hacen gozar sexualmente más }\end{array}$ & 19 & $43 \%$ \\
a las mujeres & $19 \%$ \\
\hline
\end{tabular}

Fuente: Encuesta a estudiantes de obstetricia y enfermería. validado del "Proyecto Integrado: La salud sexual y la salud reproductiva de la población estudiantil de la Universidad Nacional de Costa Rica". Instituto de Estudios Sociales en Población (IDESPO) publicado por el Instituto de Estudios Sociales en Población de la Universidad Nacional de la Facultad de Ciencias Sociales del mencionado país ${ }^{(10)}$.

Para la recolección de la información se elaboró una base de datos; y para el análisis de sus resultados entre las variables, se emplearon indicadores de estadísticas descriptiva (proporciones) y analítica como el valor de $\mathrm{p}(<0,05)$ donde se comparó entre estudiantes de las escuelas de Enfermería y Obstetricia los conocimientos y actitudes acerca de salud sexual y observar la probabilidad de diferencias aplicando el test $\mathrm{T}$, para el cual se utilizó el programa Excel Microsoft Office 2010 en español.

\section{RESULTADOS}

Del total de los encuestados, se observó que el $64 \%$ que corresponden a estudiantes de obstetricia y $36 \%$ pertenecen a enfermería. En relación al sexo del total de estudiantes encuestados el $74 \%$ son de sexo femenino mientras que el $26 \%$ son de sexo masculino. En relación al estado civil se observó que eran solteros(as) $79 \%$, en unión libre $10 \%$ y casados(as) $6 \%$. En cuanto a números de hijos, no tienen hijos el 79\%, tienen un hijo $16 \%$, dos hijos $3 \%$, y más de dos hijos el $2 \%$; existiendo una mayor proporción de estudiantes que no tienen hijos.

En relación a la creencia religiosa que profesan los estudiantes, expresaron ser evangélicos el 13\%, de religión pentecostal el 2\%, católica el 39\%, testigos de Jehová el $17 \%$, mormona $7 \%$, otras religiones $3 \%$, ninguna religión $1 \%$, y son ateos 18\%; obteniéndose predominio en la católica; siendo esto es coherente con el gran porcentaje de catolicismo del país. Además, de acuerdo a la religiosidad, la misma que se consultó de manera directa, dijeron ser muy religiosos el 10\%, regularmente religiosos el $47 \%$, poco religiosos el $28 \%$, nada religioso el $15 \%$, observándose que existe un predominio de regularmente religioso. (Tabla 1 ) 
En relación al conocimiento del tema de salud sexual, el nivel es crítico. Ante preguntas que dicen no saber, como si la sexualidad es solo lo biológico o anatómico, refieren no saber lo mismo se observa cuando mencionan que los jóvenes no tiene preparación suficiente para los aspectos relacionales y emocionales de una relación intima; asimismo se observa que no distinguen entre los deseos personales y los de otra persona, estos conceptos generalmente deberían haberse plasmado en etapa colegial; sin embargo, lo más preocupante es cuando el $5 \%$ de los entrevistados refiere que el sexo es mejor cuando se toma droga. (Tabla 2)

En cuanto a las actitudes hacia la salud sexual, el $50 \%$ de los estudiantes está en desacuerdo en que los hombres que se arreglan más sean afeminados, y el $81 \%$ desaprueba que las mujeres que no se preocupan por su apariencia sean marimachos. El 25\% de entrevistados manifiesta a la masturbación como exclusiva de los hombres. El 12\% manifiesta que la mujer vale más cuando es virgen y el $14 \%$ que las relaciones prematrimoniales sean perjudiciales para la mujer. Esto se puede interpretar generalmente por la parte cultural y de valores que vienen recibiendo dentro del hogar; por otro lado, $66 \%$ piensan que las relaciones sexuales deben darse solamente cuando hay amor, un pensamiento muy abstracto ya que dentro de las prácticas reflejan otra situación. (Tabla 3)

Referente a prácticas relacionadas a la salud sexual, el 92\% se declara como persona sexualmente activa, mientras que el $76 \%$ ha practicado sexo oral. Esto puede evaluarse en el contexto que existe un comportamiento de riesgo en la población estudiada, por lo cual hay que concientizar inmediatamente a todos los estudiantes de la facultad de Ciencias de la salud. (Tabla 4)

En general, los estudiantes presentaron un nivel medio en conocimientos y en comportamiento; que al comparar entre estudiantes de las escuelas de Enfermería y Obstetricia, se obtuvo que en los universitarios de la carrera de obstetricia tengan un promedio mayor en conocimientos y actitudes acerca de salud sexual. Se encontró diferencia significativa entre los varones y mujeres de enfermería en rela-

Tabla 3. Actitudes hacia la Salud Sexual.

\begin{tabular}{lcc}
\hline Descripción & No. & Porcentaje \\
\hline Los hombres que se preocupan por su apariencia física son "afeminados" & 17 & 25 \\
\hline La masturbación la practican exclusivamente los hombres & 17 & 25 \\
\hline $\begin{array}{l}\text { Las mujeres que no se preocupan por su apariencia física son "marimachos" 0 } \\
\text { "tortilleras" }\end{array}$ & 33 & 47 \\
\hline Me siento presionado(a) por mis amistades para tener relaciones sexuales & 24 & 34 \\
\hline La mujer vale más cuando es virgen & 8 & 12 \\
\hline El hombre vale más cuando es virgen & 6 & 9 \\
\hline Las relaciones sexuales prematrimoniales son perjudiciales para las mujeres & 10 & 14 \\
\hline Las relaciones sexuales prematrimoniales son perjudiciales para los hombres & 5 & 7 \\
\hline Masturbarse es pecado & 3 & 2 \\
\hline El hombre necesita las relaciones sexuales más que la mujer & 10 & 14 \\
\hline Voy a tener relaciones sexuales hasta que me case & 78 & 56 \\
\hline El acto sexual siempre debe culminar con la penetración & 89 & 64 \\
\hline Preferíía que mi pareja sea más experimentada que yo & 61 & 44 \\
\hline Siento temor de tener sexo porque puedo adquirir una infección de transmisión & 91 & 65 \\
\hline sexual & 70 & 50 \\
\hline Siento temor de tener sexo porque puede resultar en un embarazo & 70 \\
\hline
\end{tabular}

Fuente: Encuesta a estudiantes de obstetricia y enfermería.

Tabla 4. Prácticas relacionadas con la Salud Sexual.

\begin{tabular}{lcc} 
Descripción & No. & Porcentaje \\
\hline Practico el sexo oral & 106 & 76 \\
\hline Me masturbo & 49 & 35 \\
$\begin{array}{l}\text { Soy una persona sexualmente } \\
\text { activa }\end{array}$ & 129 & 92 \\
\hline
\end{tabular}

Fuente: Encuesta a estudiantes de obstetricia y enfermeria

ción a prácticas en salud sexual; además, se encontró diferencia entre hombres y mujeres acerca de sus prácticas en salud sexual en obstetricia, siendo de más puntaje las mujeres. (Tabla 5)

\section{DISCUSIÓN}

Se discutieron las respuestas de cada dimensión en función de los conocimientos y su comportamiento de los estudiantes que participaron en este estudio. Se observó que el $92 \%$ se declara como persona sexualmente activa, mientras que el $76 \%$ ha practicado sexo oral atribuyendo que existe un comportamiento de riesgo en la población estudiada que es similar al estudio realizado en universitarios de Cali - Colombia, en donde la mayoría de los estudiantes asumen riesgos asociados al no uso de medidas preventivas cuando asocian confianza y estabilidad en la relación de pareja, sin embargo, se reportaron múltiples parejas sexuales por año, aspecto que aumenta la posibilidad de contraer una ITS. ${ }^{(11)}$ La presente investigación es muy similar al estudio en educandos de la Facultad de Tecnología de la Salud del Municipio Cerro, donde el 91\% de los encuestados tenía vida sexual activa; además, se reportó que el $27 \%$ había tenido más de una pareja al año y el 11,0 \% más de una pareja a la vez. ${ }^{(12)}$

En lo que se refiere a salud sexual se aprecia que los conocimientos en preguntas verdaderas las mujeres respondieron mejor que los varones $32 \%$ versus el $25 \%$ respectivamente: en las preguntas falsas hubo un porcentaje considerable que no respondió bien (40\% varones versus el 33\% de las mujeres). Al comparar nuestros resultados con otros estudios realizados en Cali Colombia en la revista virtual de la Universidad Católica del Norte en el año 2009 cuyo artículo conductas sexuales de riesgo en estudiantes universitarios de la ciudad de Cali de Ana Fernanda Uribe Rodríguez, donde los niveles de conocimientos de los estudiantes universitarios eran muy bajos, ya que 
Tabla 5. Puntaje de conocimientos actitudes y prácticas en salud sexual según escuela universitaria.

\begin{tabular}{|c|c|c|c|c|c|c|c|}
\hline \multirow{2}{*}{\multicolumn{2}{|c|}{ Facultad a la que pertenece / Sexo }} & \multicolumn{2}{|c|}{ Conocimientos } & \multicolumn{2}{|c|}{ Actitudes } & \multicolumn{2}{|c|}{ Practicas } \\
\hline & & Hombres & Mujeres & Hombres & Mujeres & Hombres & Mujeres \\
\hline Enfermería & ${ }^{* * *} P$ & 13 & 11,4 & 59,4 & 55,5 & 5,1 & 7,4 \\
\hline Obstetricia & ${ }^{* * *} A * * * P$ & 13 & 13,6 & 61,8 & 64 & 6 & 8,1 \\
\hline
\end{tabular}

${ }^{* * *}$ A Diferencia estadistica significativa $(p: 0,05)$ entre los géneros en las actitudes.

***P Diferencia estadística significativa $(p: 0,05)$ entre los géneros en las prácticas.

Fuente: Encuesta a estudiantes de obstetricia y enfermería.

el 20,2\% recibían conocimientos sobre sexualidad de su universidad y el resto lo obtenían de los medios televisivos, internet, entre otras. ${ }^{(13)}$ los resultados de esta investigación se los podría vincular con lo que señalan Stern, Fuentes, Zurita, Lozano Treviño y Reysoo en el año 2003 cuando mencionan que los jóvenes no tienen preparación suficiente para los aspectos relacionales y emocionales de la relación íntima en cuanto a conocimiento. ${ }^{(14)}$

Respecto al comportamiento en salud sexual, los resultados demostraron niveles medios a bajos en la población estudiada, siendo la mayoría de los estudiantes varones (quienes piensan y opinan según valores aprendidos en el hogar o en la calle) no respetar la sexualidad de la mujer y el cuidado que amerita la protección en el uso del condón en las relaciones sexuales. Otro aspecto importante de mencionar es que la mayoría de las estudiantes mujeres ya han iniciado relaciones sexuales (78\%), resultados relacionados con otros estudios como el que se hizo en el año 2014 (cuyo tema fue conocimientos y actitudes respecto a la sexualidad en los estudiantes de la facultad de ciencias sociales de HTR Carranza) en que se evidenció dentro del grupo de estudiantes universitarios encuestados que a menor conocimiento el comportamiento será de mayor riesgo. ${ }^{(15)}$ También se podría vincular este estudio con similitudes en el comportamiento sexual de riesgo ETS/SIDA en adolescentes en el departamento de Lambayeque del autor Víctor Soto Cáceres, en la cual la mayoría de adolescentes habían tenido ya una relación sexual. ${ }^{(16)}$

Los resultados de la investigación están circunscritos a la población y período de estudio. Si bien este estudio presenta las bondades en el conocimiento de la salud sexual en estudiantes universitarios, pero considerando que solo es sobre sa- lud sexual, sería conveniente incorporar la salud reproductiva, además ampliar el universo a otras carreras universitarias que permita la aplicación del enfoque y comparación del estudio.

Por lo que se concluye en forma general, que los estudiantes mayormente se declaran sexualmente activos y tres de cada cuatro estudiantes practican el sexo oral, presentando un nivel medio en conocimientos y comportamiento en relación a salud sexual. Cuando se compara entre facultades se observa que los jóvenes de Obstetricia tienen un promedio mayor en conocimientos y actitudes acerca de salud sexual. Se encontró diferencia significativa entre los hombres y mujeres de enfermería acerca de sus prácticas en salud sexual. También se encontró diferencia acerca de sus actitudes y prácticas en obstetricia, siendo las mujeres las de mejor puntuación.

\section{REFERENCIAS BIBLIOGRÁFICAS}

1. Rodriguez A, Sanabria G, Contreras M, Perdomo B. Estrategia educativa sobre promoción en salud sexual y reproductiva para adolescentes y jóvenes universitarios. Rev Cuba Salud Pública. 2013;39(1):161-74. Disponible en: http:// scielo.sld.cu/scielo.php?script=sci_arttext\&pid =S0864-34662013000100015

2. Águila $Y$, Reyes $H$, Enrique $V$, Castro $H$, Higinio $V$. Las consecuencias de la violencia de género para la salud y formación de los adolescentes. Rev Médica Electrónica. 2016;38(5):697-710. Disponible en: http://scielo.sld.cu/scielo.php?pid=S1684 $18242016000500005 \&$ script=sci_arttext\&tlng=pt

3. Fernández L, Rodríguez Y, Sori N. Actividades educativas dirigidas a la preparación de las familias para la educación sexual de los adolescentes con diagnóstico de retraso mental moderado. Cuad Educ Desarro [Internet]. 2013 [citado 15 de marzo de 2017]; (33). Disponible en: http://atlante.eumed. net/wp-content/uploads/actividades-educativas.pdf

4. Fernández A. La educación sexual, una asignatura pendiente en España. Bio-Graf [Internet]. 2016 [citado 15 de marzo de 2017];9(16). Disponible en: http://revistas.pedagogica.edu.co/index.php/ bio-grafia/article/view/4510

5. Naranjo J, Augusto J, Delgado A, Rodríguez R,
Sánchez Y. Consideraciones sobre el Modelo de Atención Integral de Salud del Ecuador. Rev Cuba Med Gen Integral. 2014;30(3):375-381

http://scielo.sld.cu/scielo.php?pid=S0864$21252014000300011 \&$ script=sci_arttext\&tlng=en

6. Manual_MAIS-MSP12.12.12.pdf [Internet]. [citado 15 de marzo de 2017]. Disponible en: https:// instituciones.msp.gob.ec/somossalud/images/ documentos/guia/Manual_MAIS-MSP12.12.12.pdf

7. Malo-Serrano M, Malo-Corral N. Reforma de salud en Ecuador: nunca más el derecho a la salud como un privilegio. Rev Peru Med Exp Salud Publica. 2014;31(4):754-61. http://www.scielo.org.pe/scielo. php?pid=S1726-46342014000400022\&script=sci_ arttext\&tlng $=p t$

8. Hernández R, Fernández $C$, Valencia $M$, Torres $S$, Sampieri C, Fernández M, et al. Metodologia de la investigación [Internet]. McGraw-Hill Education,; 2014 [citado 15 de marzo de 2017]. Disponible en: http://www.sidalc.net/cgi-bin/wxis. exe/?IsisScript=UCC.xis\&B1 $=$ Buscar\&formato $=$ 1 \& cantidad $=50$ \&expresion $=$ Hern $\%$ E1 ndez $\% 20$ Sampieri,\%20Roberto,

9. Sabino C. El proceso de investigación [Internet]. Editorial Episteme; 2014 [citado 15 de marzo de 2017]. Disponible en: https://books.google.es/bo oks?hl=es\&lr=\&id=jwejBAAAQBAJ\&oi=fnd\&pg=P $\mathrm{P} 6 \& d q=$ metodologia + de + la + investigacion $+\& o t s=$ WNigxM1cWF\&sig=2ZsypZFgddLPVaNwNkje25V 2Cmk

10. Ruiz R. Documento: Conocimientos, actitudes y prácticas en salud sexual y salud reproductiva: Propuesta de una escala psicométrica. 21 de agosto de 2013 [citado 15 de marzo de 2017]; Disponible en: http://repositorio.una.ac.cr/handle/11056/7360

11. Orcasita L, López M, Gómez C. Conocimientos sobre riesgos frente a infecciones de transmisión sexual (ITS) en estudiantes universitarios de la ciudad de Cali. Inf Psicológicos. 2014;14(1):143-158. http://revistas.upb.edu.co/index.php/informespsicologicos/article/view/3079

12. Cortés A, Garcia R, Ochoa R. Comportamiento sexual y uso del condón en estudiantes de la facultad de tecnologia de la salud. Rev Cubana Med Trop. 2015;67(2):202-212. Disponible en: http://scielo.sld.cu/scielo.php?pid=S037507602015000200007\&script=sci_arttext\&tIng=en

13. Hernández S, Carmen R del, Pérez Piñero J, Sanabria Ramos G. Vivencias de la sexualidad en estudiantes universitarios. Rev Cuba Salud Pública. 2013;39:915-28. Disponible en: http:// scielo.sld.cu/scielo.php?script=sci_arttext\&pid =S0864-34662013000500010

14. Delgado M, Gamiño S, Delgado K, Cisneros C, Barroso F, Santos V. Rasgos de personalidad de escolares adolescentes en entorno conflictivo. Acta Univ. 2014;24(2):61-6. Disponible en: https:// scholar.google.es/scholar?q=Rasgos+de+persona lidad+de+escolares+adolescentes+en+entorno+c onflictivo\&btnG=\&hl=es\&as_sdt=0\%2C5

15. Carranza $H$, Lino $O$, Fabián $M$, Damián $A$, Lino $E$, Gamarra $\mathrm{H}$, et al. Conocimiento y actitudes respecto a la sexualidad en los estudiantes de la Facultad de Ciencias Sociales 2014. INFINITUM. [Internet]. 3 de enero de 2016 [citado 28 de mayo de 2016];5(02). Disponible en: http://web.unjfsc.edu.pe/index.php/ Infinitum/article/view/311

16. Soto Cáceres V. Comportamiento sexual de riesgo para ETS/SIDA en adolescentes del departamento de Lambayeque. Folia Dermatol Perú. 1998;9(1/2):22-8. http://scielo.sld.cu/scielo.php?script=sci_arttext\&p id=S0864-34662013000500010 